\title{
Mathematical study on a dynamical predator-prey model with constant prey harvesting and proportional harvesting in predator
}

Md Golam Mortuja ( $\nabla$ mdgolam@iiitnr.edu.in )

IIIT-NR: Dr Shyama Prasad Mukherjee International Institute of Information Technology https://orcid.org/0000-0001-7898-3711

Mithilesh Kumar Chaube

IIIT-NR: Dr Shyama Prasad Mukherjee International Institute of Information Technology

Santosh Kumar

IIIT-NR: Dr Shyama Prasad Mukherjee International Institute of Information Technology

\section{Research Article}

Keywords: Predator-prey system, Harvesting, Square root functional response, Stability, Bifurcation

Posted Date: March 31st, 2021

DOl: https://doi.org/10.21203/rs.3.rs-377501/v1

License: (c) (1) This work is licensed under a Creative Commons Attribution 4.0 International License. Read Full License 


\title{
Mathematical study on a dynamical predator-prey model with constant prey harvesting and proportional harvesting in predator
}

\author{
Md Golam Mortuja • Mithilesh Kumar \\ Chaube · Santosh Kumar
}

Received: date / Accepted: date

\begin{abstract}
A dynamical predator-prey model with constant prey harvesting, proportional harvesting in predator has been studied. The square root functional response also has been incorporated in the system to describe the prey herd behaviour, assuming the average handling time is zero. The existence and the local stability of equilibria of the system have been discussed. It is examined that, two types of bifurcation occur in the system. The two types of bifurcations have been analyzed, and it has been found by analyzing the saddle-node bifurcation that, there is a maximum sustainable yield. It is observed that if harvesting rate is greater than the maximum sustainable yield, the prey population abolish from the system and then extinction of the predator population happen. But if harvesting rate is lesser than the maximum sustainable yield, the extinction of the prey population can not be possible. By analyzing the Hopf bifurcation, it is obtained that, there exists an unstable limit cycle around the interior equilibrium point. Several numerical simulations are performed to check the results.
\end{abstract}

Keywords Predator-prey system · Harvesting · Square root functional response $\cdot$ Stability $\cdot$ Bifurcation

\footnotetext{
Md Golam Mortuja

E-mail: mdgolam@iiitnr.edu.in

Mithilesh Kumar Chaube

E-mail: mithilesh@iiitnr.edu.in

Santosh Kumar

E-mail: santosh@iiitnr.edu.in
}

DSPM IIIT NR, CG, India 


\section{Introduction}

To describe the interactions between different species in the nature, predatorprey system is one of the most important and useful models. Lotka (1) and Voterra (2) separately offered basic models to describe the interaction between two species and proposed a two dimensional system of ordinary differential equations. They introduced a functional response and used in their model which is proportional to the density of predator. Later, many researchers studied on the classical Lotka-Volterra (1) (2) model and also produced different types of functional responses, e.g., C.S Holling (3) introduced three types of functional responses, Crowley-Martin (4), Beddington-DeAngelis (5) (6) offered different functional responses, etc. In the mentioned studies the authors used these functional responses by assuming that the predator can take any prey for their food i.e., they considered solitary prey behaviour.

But, in nature some preys live in herd behaviour to fight with the predators for their benefits. For that type of herd behaviour, the above mentioned functional responses are not appropriate to describe the interaction. To investigate the model with that type of prey herd behaviour, a model with a new functional response was proposed by Ajraldi et al.(7) and the author states that, it is more appropriate to model the functional response for prey herd behaviour as a square root function of prey density. Later, Braza in his paper(하) introduced a new functional response considering the square root of prey density in the Holling Type II functional response (3) and assuming the average handling time is zero he proposed a model with this functional response. Further many researchers studied that model to investigate the interaction between predator and prey with the functional response (9) (10) (11) (12) (13) (14). In this paper, this type of functional response has been included in the model.

Nowadays, people are very much interested to harvest the species for economic interest which very much affects on the dynamics of any ecological or biological system. For example, we can see in any marine ecosystem people are harvesting fishes for their economic interest which affects on the dynamics of a marine ecosystem. To tackle that type of situation, many researchers worked on a predator-prey system with harvesting effects (15) (16) (17) (18). Basically researchers used three types of harvesting and the classification is constant harvesting (19), proportional harvesting (20) and non-linear harvesting (21). In this paper we consider harvested predator and prey population. Prey populations are harvested by constant rate and predator populations are harvested by proportional rate. The main aim of the paper is to investigate the dynamical behaviour of a predator prey system with constant harvesting in prey, proportional harvesting in predator and also with square root functional response assuming average handling time is zero. The organization of the rest of the paper in this way:

In Sec 2 we formulate a mathematical model with constant harvesting in prey, proportional harvesting in predator and also with square root functional response assuming average handling time is zero. The condition for existence of equilibria and their local stability of system (3) have been discussed in Sec.3. 
In Sec 4 two types of bifurcations of the system (3) have been analyzed. Sec 5 includes numerical examples. Finally, Sec 6 contains the conclusion.

\section{Mathematical Model Formulation}

The basic predator-prey model with the square root functional response (8), constant harvesting in prey and proportional harvesting in predator is as follows:

$$
\left\{\begin{array}{l}
\frac{d x(t)}{d t}=r x\left(1-\frac{x}{N}\right)-\alpha \sqrt{x} y-h, \\
\frac{d y(t)}{d t}=-\beta y+m \alpha \sqrt{x} y-E_{q} y .
\end{array}\right.
$$

Where the prey population denoted by $x$ and the predator population denoted by $y, r$ is the growth rate of prey, $m$ is the biomass conversion rate, $\alpha$ denotes the predator's search efficiency for prey. $N$ is the environment carrying capacity, $h$ is the constant prey harvesting and $\beta$ is the predator's natural death rate in the prey free situation and $E_{q}$ is harvesting effort. For the dynamic analysis of the model, the variables and the parameters are scaled as, $\bar{x}=\frac{x}{N}, \bar{y}=\frac{\alpha y}{r \sqrt{N}}, \bar{t}=r t, c=\frac{m \alpha \sqrt{N}}{r}, d=\frac{\beta}{r}, E=E_{q} \frac{\sqrt{N}}{\alpha}$

Then the system (1) becomes:

$$
\left\{\begin{array}{l}
\frac{d \bar{x}(t)}{d t}=\bar{x}(1-\bar{x})-\sqrt{\bar{x}} \bar{y}-h \\
\frac{d \bar{y}(t)}{d t}=-d \bar{y}+c \sqrt{\bar{x}} \bar{y}-E \bar{y}
\end{array}\right.
$$

Excluding the bars of the system (2) we formulate the following system:

$$
\left\{\begin{array}{l}
\frac{d x(t)}{d t}=x(1-x)-\sqrt{x} y-h, \\
\frac{d y(t)}{d t}=-d y+c \sqrt{x} y-E y .
\end{array}\right.
$$

Where $c, d, h, E$ all are positive.

\section{Existence and stability of equilibria}

3.1 Existence of equilibria

The system (3) has been defined on the following set considering biological background: $\Gamma=\left\{(x, y) \in \mathbb{R}^{2} ; x \geq 0, y \geq 0\right\}$.

To identify the equilibrium points of the system (3), the following system has 
been solved:

$$
\left\{\begin{aligned}
x(1-x)-\sqrt{x} y-h & =0 \\
-d y+c \sqrt{x} y-E y & =0 .
\end{aligned}\right.
$$

The above system has three equilibrium points and we get the following theorems regarding their existence.

Note that:

$$
\left\{\begin{array}{c}
x_{1}=\frac{1+\sqrt{1-4 h}}{2}, x_{2}=\frac{1-\sqrt{1-4 h}}{2}, \\
x_{*}=\left(\frac{d+E}{c}\right)^{2}, y_{*}=\sqrt{x_{*}}\left(1-x_{*}\right)-\frac{h}{\sqrt{x_{*}}}
\end{array}\right.
$$

\section{Theorem 1 [(i)]}

When $0<4 h<1$, the system (3) has two axial equilibrium points $E_{i}=$ $\left(x_{i}, 0\right),(i=1,2)$, which are different.

๑. When $4 h=1$, two equilibria collide and produce a unique axial equilibrium point $E_{0}=\left(x_{0}, 0\right)=\left(\frac{1}{2}, 0\right)$.

3. When $\sqrt{x_{*}}\left(1-x_{*}\right)-\frac{h}{\sqrt{x_{*}}}>0$, then the equilibrium point of the system (3) exists uniquely which is denoted by $E_{*}=\left(x_{*}, y_{*}\right)$.

Proof. If $4 h>1$, then $\frac{d x(t)}{d t}=x(1-x)-\sqrt{x} y-h<0$ in $\Gamma$. This implies that prey population will go extinct and then extinction of predator population will happens. It is obvious that in $\Gamma$ the system (3) has no equilibria. Now for predator free (i.e., $\mathrm{y}=0$ ) equilibrium points we have solved the following quadratic equation:

$$
x^{2}-x+h=0 .
$$

Two different roots $x_{1}, x_{2}$ of the equation (6) are mentioned in (6) are positive when $4 h<1$ for positiveness of the equilibrium points. And if $4 h=1$, two equilibria collide and the unique value is $\frac{1}{2}$. For interior equilibrium point we solve the system (4). From second equation of the system (4) we get, $\sqrt{x_{*}}=\left(\frac{d+E}{c}\right)$, and after putting the value of $x_{*}$ from first equation of the system 44 we get, $y_{*}=\sqrt{x_{*}}\left(1-x_{*}\right)-\frac{h}{\sqrt{x_{*}}}>0$.

\subsection{Stability of equilibria}

This subsection includes the dynamic analysis of the system (3) around the equilibrium points. The following matrix $J$ is the Jacobian matrix of the system 
(3):

$$
J=\left(\begin{array}{cc}
-2 x-\frac{y}{2 \sqrt{x}}+1 & -\sqrt{x} \\
\frac{c y}{2 \sqrt{x}} & c \sqrt{x}-(d+E)
\end{array}\right) .
$$

Now, the local stability of the system (3) are analyzed in the neighbourhood of $E_{1}$ and $E_{2}$. And regarding this we have the following theorems.

Theorem 2 When $4 h<1$ the system (3) has two different equilibria $E_{1}$ and $E_{2}$.

[(i)]If $c \sqrt{x_{1}}>(d+E)$, then $E_{1}$ is hyperbolic saddle. If $c \sqrt{x_{1}}<(d+E)$, then $E_{1}$ is stable.

Proof. It can be seen that from Theorem (1) that, the system (3) has two different equilibria $E_{1}$ and $E_{2}$. Now the following matrix $J\left(E_{1}\right)$ is the Jacobian matrix at $E_{1}$ of the system 3

$$
J\left(E_{1}\right)=\left(\begin{array}{cc}
1-2 x_{1} & -\sqrt{x_{1}} \\
0 & c \sqrt{x_{1}}-(d+E)
\end{array}\right) .
$$

The eigenvalues $\lambda_{1}, \lambda_{2}$ of the Jacobian matrix $J\left(E_{1}\right)$ are as follows:

$\lambda_{1}=1-2 x_{1}=-\sqrt{1-4 h}<0$, since $4 h<1$,

$\lambda_{2}=c \sqrt{x_{1}}-(d+E)>0$, if $c \sqrt{x_{1}}>(d+E)$. Hence, $E_{1}$ is hyperbolic saddle. Now, if $c \sqrt{x_{1}}<(d+E)$, then $\lambda_{2}=c \sqrt{x_{1}}-(d+E)<0$. Hence, $E_{1}$ is stable.

Q. Theorem 3 When $4 h<1$ the system (3) has two different equilibria $E_{1}$ and $E_{2}$.

[(i)]If $c \sqrt{x_{2}}>(d+E)$, then $E_{2}$ is unstable. If $c \sqrt{x_{1}}<(d+E)$, then $E_{2}$ is hyperbolic saddle.

Proof. From Theorem (1) it can be observed that, the system (3) has two different equilibria $E_{1}$ and $E_{2}$. The following matrix $J\left(E_{2}\right)$ is the Jacobian matrix at $E_{2}$ of the system (3)

$$
J\left(E_{2}\right)=\left(\begin{array}{cc}
1-2 x_{2} & -\sqrt{x_{2}} \\
0 & c \sqrt{x_{2}}-(d+E)
\end{array}\right) .
$$

The eigenvalues $\mu_{1}, \mu_{2}$ of the Jacobian matrix $J\left(E_{2}\right)$ are as follows: $\mu_{1}=1-2 x_{2}=\sqrt{1-4 h}>0$, since $4 h<1$,

$\mu_{2}=c \sqrt{x_{2}}-(d+E)>0$, if $c \sqrt{x_{2}}>(d+E)$. Hence, $E_{2}$ is unstable. If $c \sqrt{x_{2}}<(d+E)$, then $\mu_{2}=c \sqrt{x_{2}}-(d+E)<0$. Hence, $E_{1}$ is hyperbolic saddle.

Now, the local stability of the unique interior equilibrium point $E_{*}$ are analyzed and the following theorem is obtained.

Q. Theorem 4 When $\sqrt{x_{*}}\left(1-x_{*}\right)-\frac{h}{\sqrt{x_{*}}}>0$, then there exists a unique equilibrium point $E_{*}=\left(x_{*}, y_{*}\right)$ of the system (3).

[(i)]If $h=h_{1}$, then $E_{*}$ is center. $E_{*}$ is source at $h>h_{1}>0 . E_{*}$ is sink at $h<h_{1}$. 
Note: $h_{1}=3 x_{*}^{2}-x_{*}$

Proof. Theorem 11 shows that, when $\sqrt{x_{*}}\left(1-x_{*}\right)-\frac{h}{\sqrt{x_{*}}}>0$, then there exists a unique interior equilibrium point $E_{*}=\left(x_{*}, y_{*}\right)$ of the system (3). The following matrix $J\left(E_{*}\right)$ is the Jacobian matrix at $E_{*}$ of the system (3)

$$
J\left(E_{*}\right)=\left(\begin{array}{cc}
1-2 x_{*}-\frac{y_{*}}{2 \sqrt{x_{*}}} & -\sqrt{x_{*}} \\
\frac{c y_{*}}{2 \sqrt{x_{*}}} & 0
\end{array}\right) .
$$

$\operatorname{Trace}\left(J\left(E_{*}\right)\right)=-\frac{y_{*}}{2 \sqrt{x_{*}}}-2 x_{*}+1$ and $\operatorname{Det}\left(J\left(E_{*}\right)\right)=\frac{c y_{*}}{2}>0$. Now, Trace $\left(J\left(E_{*}\right)\right)$ $=-\frac{y_{*}}{2 \sqrt{x_{*}}}-2 x_{*}+1=-\frac{\sqrt{x_{*}}\left(1-x_{*}\right)-\frac{h}{\sqrt{x_{*}}}}{2 \sqrt{x_{*}}}+1-2 x_{*}=\frac{-\left(3 x_{*}^{2}-x_{*}\right)+h}{2 x_{*}}$. Taking $h_{1}=3 x_{*}^{2}-x_{*}$, we get, $\operatorname{Trace}\left(J\left(E_{*}\right)\right)=\frac{-h_{1}+h}{2 x_{*}}$.

Now, $E_{*}$ is center when $h=h_{1}$, since the Trace of the Jacobian matrix 10 is zero at $h=h_{1}$.

$E_{*}$ is source when $h>h_{1}>0$, since the Trace of the Jacobian matrix $10 p$ is greater than zero at $h>h_{1}>0$.

$E_{*}$ is sink when $h<h_{1}$, since the Trace of the Jacobian matrix 10 is less than zero at $h<h_{1}$

\section{Bifurcation Analysis}

Form Theorem (1) and Theorem (4), it can be seen that the system (3) may exhibits saddle-node and Hopf bifurcation. So, in this section, the conditions for two different types of bifurcations have been analyzed.

\subsection{Saddle-node bifurcation}

From Theorem (1), it is observed that two equilibria of the system (3) collide and produce a unique equilibria point $E_{0}=\left(\frac{1}{2}, 0\right)$. Now, the following matrix $J\left(E_{0}\right)$ is the Jacobian matrix at $E_{0}$ of the system (3)

$$
J\left(E_{0}\right)=\left(\begin{array}{cc}
0 & -\sqrt{\frac{1}{2}} \\
0 & c \sqrt{\frac{1}{2}}-(d+E)
\end{array}\right) .
$$

Easily it can be seen that the above Jacobian matrix (11) has a zero eigenvalue. This means that, at $E_{0}$ the stability analysis of the system (3) is not possible 

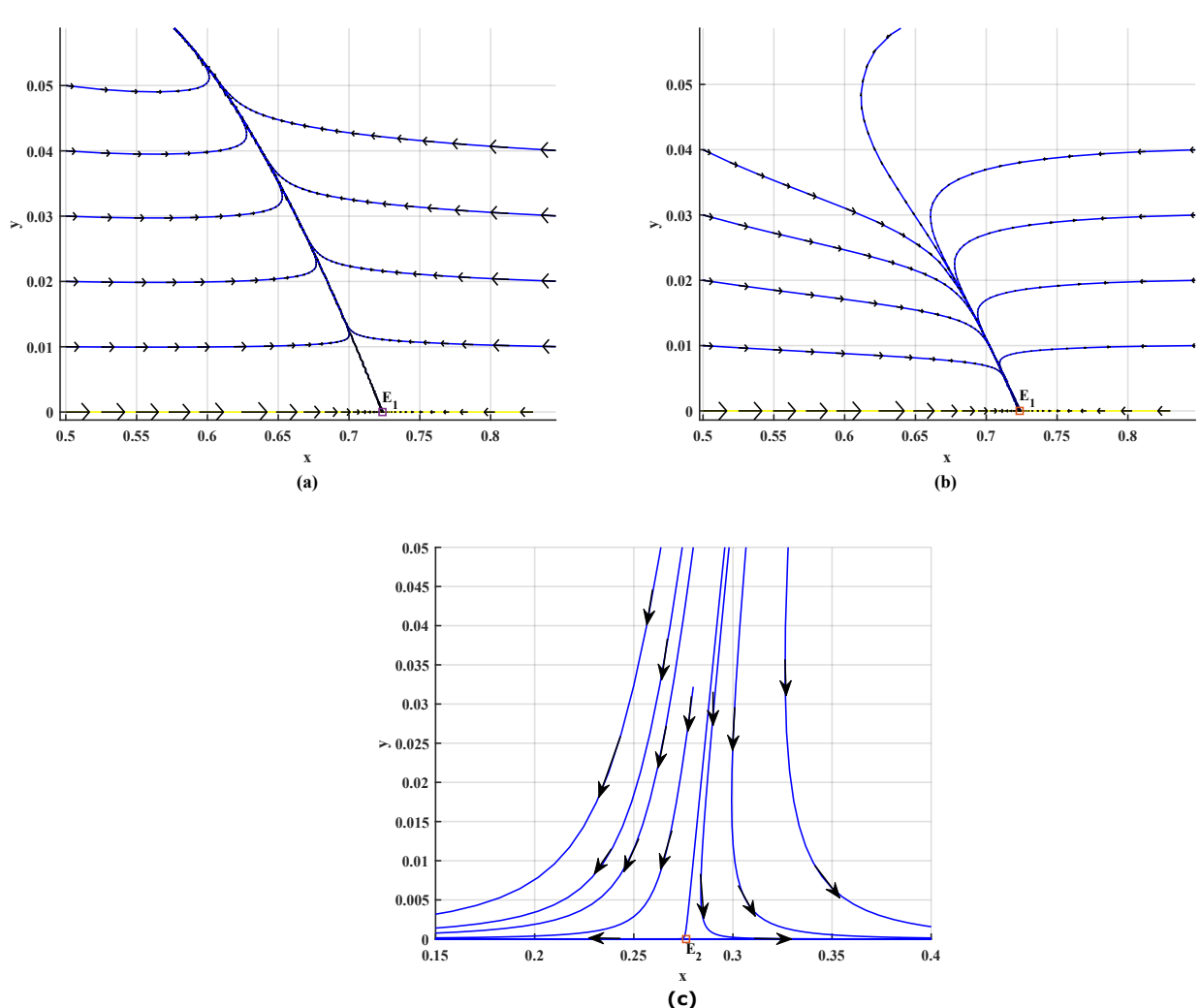

Fig. 1: (a) The Phase plane diagram of the system (3) when $c=0.2, h=$ $0.2, d=0.1, E=0.05$, where $E_{1}$ is saddle. (b) The Phase plane diagram of the system (3) when $c=0.2, h=0.2, d=0.1, E=0.1$, where $E_{1}$ is stable. (c) The Phase plane diagram of the system (3) when $c=0.5, h=0.2, d=0.6, E=0.6$, where $E_{2}$ is saddle.

by linearization technique. Therefore, the system (3) exhibits a saddle-node bifurcation in the neighbourhood of $E_{0}$, as two equilibria collide at the bifurcation parameter $h=\frac{1}{4}$. The following theorem describe the saddle-node bifurcation.

\$. Theorem 5 A saddle-node bifurcation occurs in the system (3) around $E_{0}$ at the bifurcation point $h=\frac{1}{2}$, when $4 h=1$.

Proof. As $J\left(E_{0}\right)$ contains a zero eigenvalue, according to Sotomayor's theorem( $(\overline{22})$ the necessary condition for saddle-node bifurcation around $E_{0}$ at $h=\frac{1}{4}$ satisfies. We consider $U, V$ as the eigenvectors of the matrix $J\left(E_{0}\right)$ and $J\left(E_{0}\right)^{T}$ corresponding to the zero eigenvalues, respectively. 

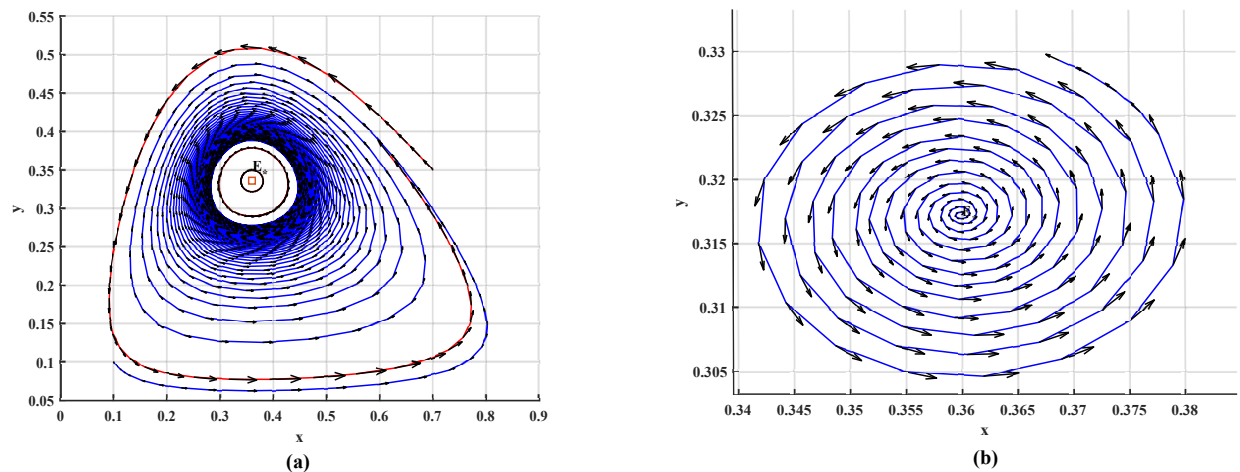

(b)

Fig. 2: (a) Phase plot of the system (3) for $c=1, h=0.0288=h_{1}, d=E=0.3$, where $E_{*}$ is center. (b) Phase plot of the system (3) for $c=1, h=0.0400>$ $h_{1}, d=E=0.3$, where $E_{*}$ is source.
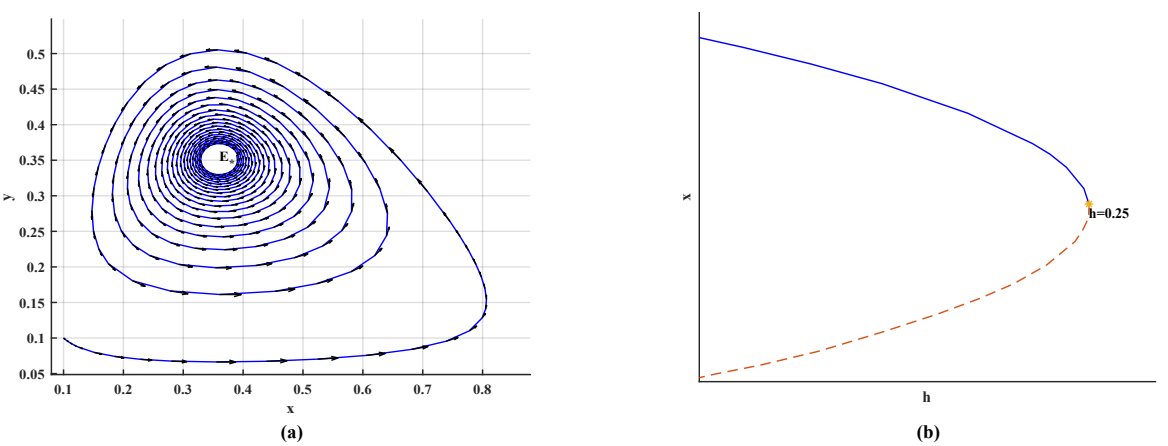

Fig. 3: (a) Phase plot of the system (3) for $c=1, h=0.0288=h_{1}, d=E=0.3$, where $E_{*}$ is sink. (b) The diagram for saddle-node bifurcation of system (3) at $h=0: 4225$ : Stable equilibrium points are represented by the solid blue curve and unstable equilibrium points are represented by green dashed curve.

$U=\left(\begin{array}{l}U_{1} \\ U_{2}\end{array}\right)=\left(\begin{array}{l}1 \\ 0\end{array}\right), W=\left(\begin{array}{l}V_{1} \\ V_{2}\end{array}\right)=\left(\begin{array}{c}c \sqrt{\frac{1}{2}}-(d+E) \\ \sqrt{\frac{1}{2}}\end{array}\right)$.

Now, we can get $g_{h}\left(E_{0}, h\right)=\left(\begin{array}{c}-1 \\ 0\end{array}\right)$ at $h=\frac{1}{4}$,

$D^{2} g_{h}\left(E_{0}, h\right)(U, U)=\left(\begin{array}{c}-2 \\ 0\end{array}\right)$. It is obvious that, $U$ and $V$ satisfy the transversality conditions: 
$V^{T} g_{h}\left(E_{0}, h\right)=(d+E)-c \sqrt{\frac{1}{2}} \neq 0$,

$V^{T} D^{2} g_{h}\left(E_{0}, h\right)=2\left[(d+E)-c \sqrt{\frac{1}{2}}\right] \neq 0$, for saddle-node bifurcation existence in the system (3) at the bifurcation parameter $h(22)$. Biologically we can conclude that, there is a maximum sustainable yield $h_{M S Y}=\frac{1}{4}$ and if $h>h_{M S Y}$, the prey population will go extinct and it will causes the extinction of predator population. But if $0<h<\frac{1}{4}$, the prey population do not go extinct.

\subsection{Hopf bifurcation}

Theorem (1) shows that, Hopf bifurcation may occur in the system (3). Now we get $\frac{d}{d h} \operatorname{Trace}\left(J\left(E_{*}\right)\right)=\frac{1}{2 x_{*}} \neq 0$ which implies that the transversality condition satisfies for occurring Hopf bifurcation. Hence, Hopf bifurcation occur in the system (3) around $E_{*}$ at $h=h_{1}(22)$. Now, the equilibrium point $E_{*}$ is translated to origin to find the Hopf bifurcation direction. Using the transformation $(X, Y)=\left(x-x_{*}, y-y_{*}\right)$ and expanding the system (3) in a power series around origin, we get

$\frac{d X}{d t}=a_{10} X+a_{01} Y+a_{11} X Y+a_{20} X^{2}+a_{02} Y^{2}+a_{21} X^{2} Y+a_{12} X Y^{2}+a_{30} X^{3}+$ $a_{03} Y^{3}+P(X, Y)$

$\frac{d Y}{d t}=b_{10} X+b_{01} Y+b_{11} X Y+b_{20} X^{2}+b_{02} Y^{2}+b_{21} X^{2} Y+b_{12} X Y^{2}+b_{30} X^{3}+$ $b_{03} Y^{3}+Q(X, Y)$,

where $P(X, Y)$ and $Q(X, Y)$ are minimum four order smooth functions of $\mathrm{X}$, $\mathrm{Y}$ and

$a_{10}=0, a_{01}=-\sqrt{x_{*}}, a_{11}=-\frac{1}{\sqrt{x_{*}}}, a_{20}=-2+\frac{y_{*}}{4 x_{*}^{\frac{3}{2}}}, a_{02}=a_{12}=a_{03}=$ $0, a_{21}=\frac{1}{4 x_{*}^{\frac{3}{2}}}, a_{30}=-\frac{3 y_{*}}{8 x_{*}^{\frac{5}{2}}}$.

$b_{01}=b_{03}=b_{12}=0, b_{10}=\frac{c y_{*}}{2 \sqrt{x_{*}}}, b_{11}=\frac{c}{\sqrt{x_{*}}}, b_{20}=-\frac{c y_{*}}{4 x_{*}^{\frac{3}{2}}}, b_{21}=-\frac{c}{4 x_{*}^{\frac{3}{2}}}, b_{30}=$ $\frac{3 c y_{*}}{8 x_{*}^{\frac{5}{2}}}$.

Now, we calculate first Lypunov number(22):

$l=-\frac{3 \pi}{2 a_{01} \Delta^{\frac{3}{2}}} N=\frac{3 \pi}{2 \sqrt{x_{*}} \Delta^{\frac{3}{2}}} N$

where $\Delta=\frac{(d+E) y_{*}}{2 \sqrt{x_{*}}}>0$, and $N=\frac{c y_{*}}{4 \sqrt{x_{*}}}\left(\frac{y_{*}}{2 x_{*}^{\frac{3}{2}}}+\frac{c}{\sqrt{x_{*}}}\right)+\frac{c y_{*}}{2}\left(c^{2}+\frac{y_{*}}{4 x_{*}^{\frac{5}{2}}}+\frac{9 y_{*}}{8 x_{*}^{2}}+\frac{c}{4 x_{*}}\right)>$

0 . Therefore, from the above expression of $\Delta$ and $N$ it can be conclude that $l>0$, that means the Hopf bifurcation is sub-critical at $h=h_{1}$. And a unstable limit cycle present in the system $(3)$ around $E_{*}(22)$. 


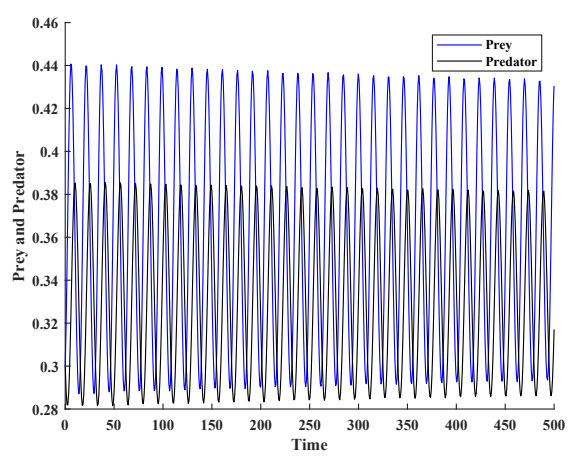

(a)

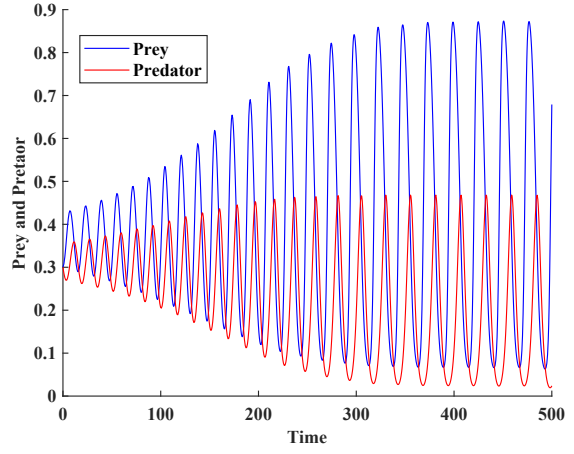

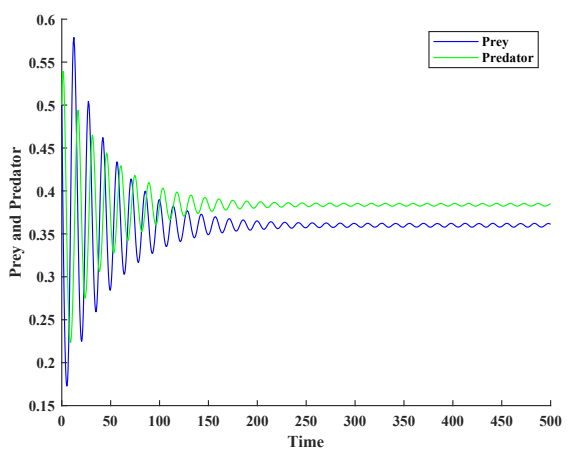

(c)

Fig. 4: (a) Time series at $h=0.0288=h_{1}$. (b) Time series at $h=0.0400>h_{1}$. (c) Time series at $h=0.0195<h_{1}$.

\section{Numerical Examples}

Some numerical examples to verify the analytical results have been discussed in this section.

- For $c=0.2, h=0.2, d=0.1, E=0.05$, the eigenvalues of $J\left(E_{1}\right)$ are $\lambda_{1}=-0.4472<0, \lambda_{2}=0.0201>0, E_{1}=(0.7236,0)$ is saddle (Figure 1a).

- For $c=0.2, h=0.2, d=0.1, E=0.1$, the eigenvalues of $J\left(E_{1}\right)$ are $\lambda_{1}=$ $-0.4472<0, \lambda_{2}=-0.0299<0, E_{1}=(0.7236,0)$ is stable (Figure $1 \mathrm{~b}$ ).

- For $c=0.5, h=0.2, d=0.05, E=0.05$, the eigenvalues of $J\left(E_{2}\right)$ are $\mu_{1}=0.4472>0, \mu_{2}=0.0051>0, E_{2}=(0.2764,0)$ is unstable. For $c=$ $0.5, h=0.2, d=0.6, E=0.6$, the eigenvalues of $J\left(E_{2}\right)$ are $\mu_{1}=0.4472>$ $0, \mu_{2}=-0.1 .0949<0, E_{1}=(0.2764,0)$ is saddle (Figure 1c).

- For $c=1, h=0.0288=h_{1}, d=E=0.3$, the Trace of $J\left(E_{2}\right)$ is zero. $E_{*}=(0.3600,0.3360)$ is center (Figure $\left.2 \mathrm{a}\right)$. 
- For $c=1, h=0.0400>h_{1}, d=E=0.3$, the Trace of $J\left(E_{2}\right)$ is $0.0156>0$. $E_{*}=(0.3600,0.3173)$ is source (Figure $\left.2 \mathrm{~b}\right)$.

- For $c=1, h=0.0195<h_{1}, d=E=0.3$, the Trace of $J\left(E_{2}\right)$ is $-0.0129<$ 0 . $E_{*}=(0.3600,0.3515)$ is sink (Figure 3a).

- The system 3 exhibit saddle-node bifurcation for $c=0.2, h=\frac{1}{4}=$ $0.25, d=0.1, E=0.05$ (Figure $3 \mathrm{~b}$ ).

\section{Conclusions}

A dynamical predator-prey model with the square root functional response to describe the prey herd behaviour assuming the average handling time is zero has been studied. Also we have incorporated constant prey harvesting, proportional harvesting in predator in the model. Conditions for the existence of the equilibria of the system (3) has been discussed. The local stability of two different predator free equilibria and a unique equilibrium point the system (3) has been analyzed. It has been observed that, two types of bifurcations occur. The saddle-node and Hopf bifurcation has been analyzed and it has been found that, there is a maximum sustainable yield $h_{M S Y}=\frac{1}{4}$. At $h>h_{M S Y}$, the prey population will go extinct and it will be the cause for extinction of the predator population. But if $0<h<\frac{1}{4}$, the prey population do not go extinct. An unstable limit cycle exists around the interior equilibrium point. It has been examined that if harvesting rate is chosen at $h \leq h_{1}$, both population will coexist and will maintained ecological balance. The calculation of first Lypunov number cleared that the Hopf bifurcation is super-critical. To enhance a good knowledge about the interaction between two species prey and predator, the results of this paper may help.

\section{References}

1. A. J. Lotka, Elements of physical biology. Williams \& Wilkins, 1925.

2. V. Volterra, "Principes de biologie mathématique," Acta biotheoretica, vol. 3, no. 1, pp. 1-36, 1937.

3. C. S. Holling, "Some characteristics of simple types of predation and parasitism," Canadian entomologist, vol. 91, no. 7, pp. 385-398, 1959.

4. P. H. Crowley and E. K. Martin, "Functional responses and interference within and between year classes of a dragonfly population," Journal of the North American Benthological Society, vol. 8, no. 3, pp. 211-221, 1989.

5. J. R. Beddington, "Mutual interference between parasites or predators and its effect on searching efficiency," The Journal of Animal Ecology, pp. 331-340, 1975.

6. D. L. DeAngelis, R. Goldstein, and R. V. O'Neill, "A model for tropic interaction," Ecology, vol. 56, no. 4, pp. 881-892, 1975. 
7. V. Ajraldi, M. Pittavino, and E. Venturino, "Modeling herd behavior in population systems," Nonlinear Analysis: Real World Applications, vol. 12, no. 4, pp. 2319-2338, 2011.

8. P. A. Braza, "Predator-prey dynamics with square root functional responses," Nonlinear Analysis: Real World Applications, vol. 13, no. 4, pp. 1837-1843, 2012.

9. S. P. Bera, A. Maiti, and G. Samanta, "Stochastic analysis of a preypredator model with herd behaviour of prey," Nonlinear Analysis: Modelling and Control, vol. 21, no. 3, pp. 345-361, 2016.

10. S. Salman, A. Yousef, and A. Elsadany, "Stability, bifurcation analysis and chaos control of a discrete predator-prey system with square root functional response," Chaos, Solitons $\&$ Fractals, vol. 93, pp. 20-31, 2016.

11. X. Zhu, Y. Dai, Q. Li, and K. Zhao, "Stability and hopf bifurcation of a modified predator-prey model with a time delay and square root response function," Advances in Difference Equations, vol. 2017, no. 1, pp. 1-15, 2017.

12. H. Liu and H. Cheng, "Dynamic analysis of a prey-predator model with state-dependent control strategy and square root response function," $A d$ vances in Difference Equations, vol. 2018, no. 1, pp. 1-13, 2018.

13. P. Chakraborty, U. Ghosh, and S. Sarkar, "Stability and bifurcation analysis of a discrete prey-predator model with square-root functional response and optimal harvesting," Journal of Biological Systems, vol. 28, no. 01, pp. 91-110, 2020.

14. P. Panja, "Combine effects of square root functional response and prey refuge on predator-prey dynamics," International Journal of Modelling and Simulation, pp. 1-8, 2020.

15. R. Gupta and P. Chandra, "Bifurcation analysis of modified leslie-gower predator-prey model with michaelis-menten type prey harvesting," Journal of Mathematical Analysis and Applications, vol. 398, no. 1, pp. 278$295,2013$.

16. P. Santra, G. Mahapatra, and D. Pal, "Analysis of differential-algebraic prey-predator dynamical model with super predator harvesting on economic perspective," International Journal of Dynamics and Control, vol. 4, no. 3, pp. 266-274, 2016.

17. K. p. Das, "A study of harvesting in a predator-prey model with disease in both populations," Mathematical Methods in the Applied Sciences, vol. 39, no. 11, pp. 2853-2870, 2016.

18. D. $\mathrm{Hu}$ and H. Cao, "Stability and bifurcation analysis in a predatorprey system with michaelis-menten type predator harvesting," Nonlinear Analysis: Real World Applications, vol. 33, pp. 58-82, 2017.

19. Y. Yao, "Bifurcations of a leslie-gower prey-predator system with ratiodependent holling iv functional response and prey harvesting," Mathematical Methods in the Applied Sciences, vol. 43, no. 5, pp. 2137-2170, 2020.

20. B. Sahoo, B. Das, and S. Samanta, "Dynamics of harvested-predatorprey model: role of alternative resources," Modeling Earth Systems and Environment, vol. 2, no. 3, pp. 1-12, 2016. 
21. S. Kumar and H. Kharbanda, "Chaotic behavior of predator-prey model with group defense and non-linear harvesting in prey," Chaos, Solitons Fractals, vol. 119, pp. 19-28, 2019.

22. L. Perko, "Differential equations and dynamical systems, texts in applied mathematics, 7," 2001.

Ethics approval (include appropriate approvals or waivers)

Consent to participate (include appropriate statements)

Consent for publication (include appropriate statements)

Availability of data and material (data transparency)

Code availability (software application or custom code)

\section{Declarations}

\subsection{Funding}

This research received no specific grant from any funding agency in the public, commercial, or not-for-profit sectors.

\subsection{Conflicts of interest}

The authors have no conflict of interest to declare.

7.3 Ethics approval

Not applicable.

\subsection{Consent to participate}

Not applicable.

7.5 Consent for publication

Not applicable.

7.6 Availability of data and material

Not applicable.

7.7 Code availability

Not applicable. 
7.8 Authors' contributions

Conceptualization: [Md Golam Mortuja, Mithilesh Kumar Chaube]; Methodology: [Md Golam Mortuja]; Formal analysis and investigation: [Md Golam Mortuja, Mithilesh Kumar Chaube, Santosh Kumar]; Writing - original draft preparation: [Md Golam Mortuja]; Supervision: [Mithilesh Kumar Chaube, Santosh Kumar]. 
Figures

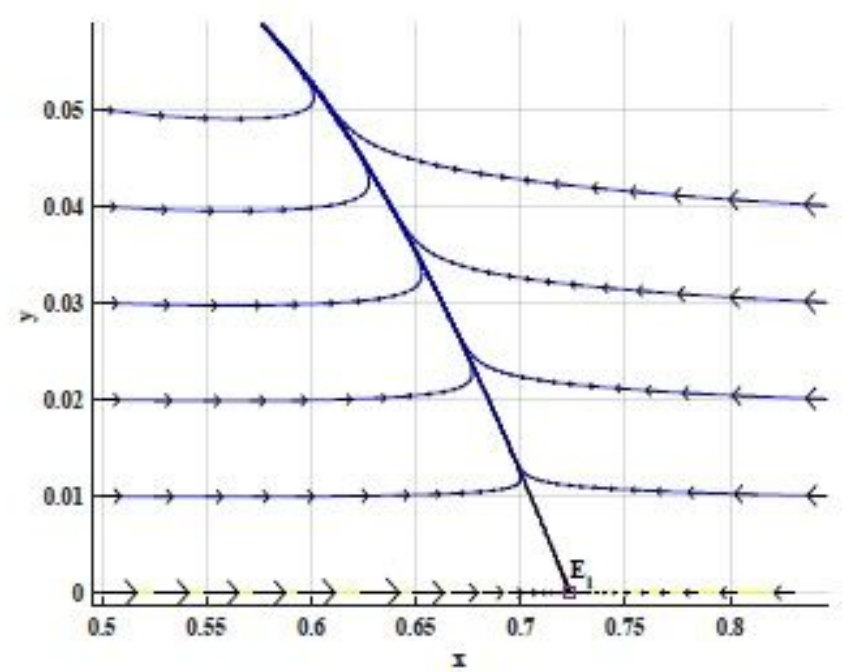

(a)

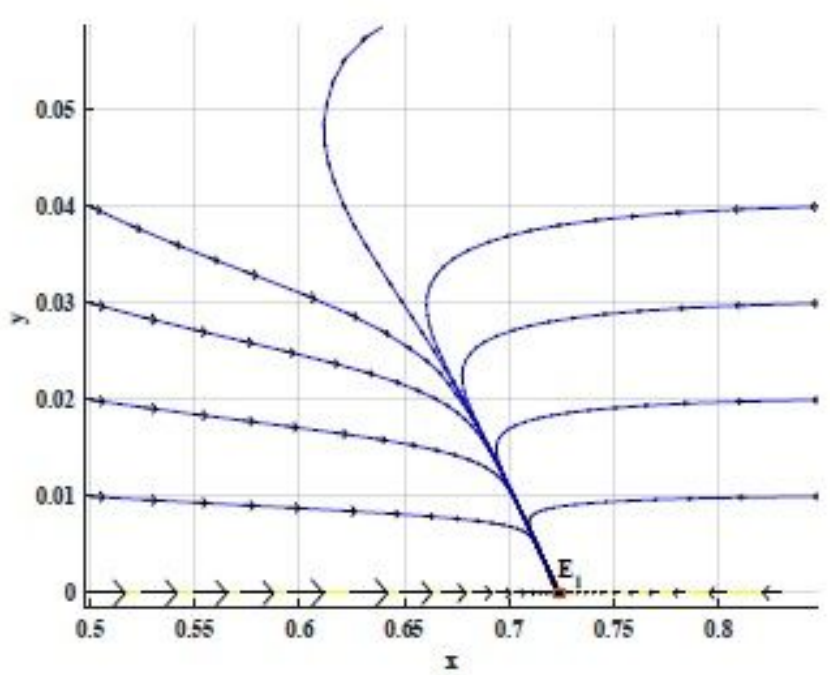

(b)

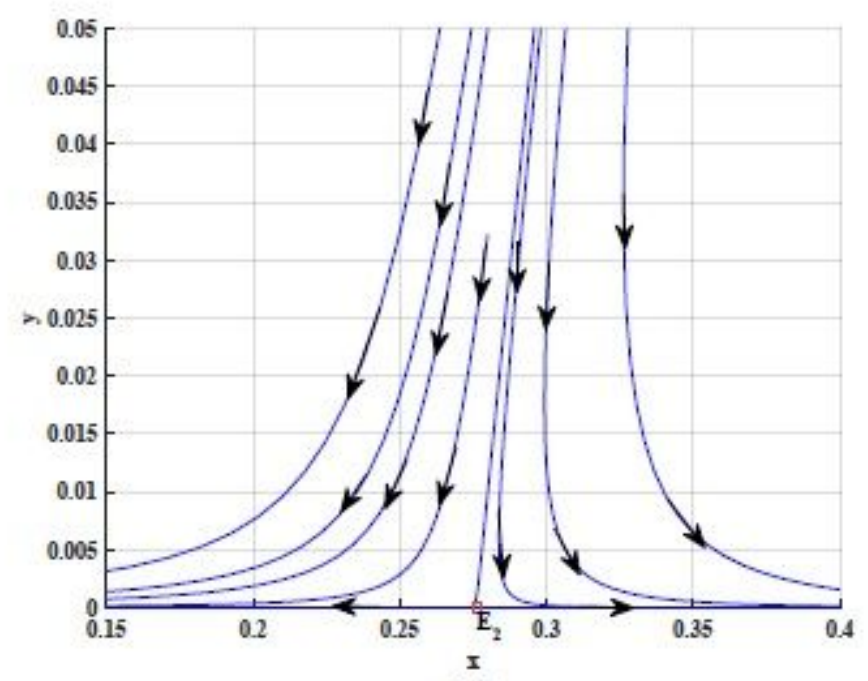

(c)

\section{Figure 1}

(a) The Phase plane diagram of the system (3) when $c=0: 2 ; \mathrm{h}=0: 2 ; \mathrm{d}=0: 1 ; \mathrm{E}=0: 05$, where $\mathrm{E} 1$ is saddle.

(b) The Phase plane diagram of the system (3) when $c=0: 2 ; h=0: 2 ; d=0: 1 ; E=0: 1$, where E1 is stable.

(c) The Phase plane diagram of the system (3) when $c=0: 5 ; h=0: 2 ; d=0: 6 ; E=0: 6$, where $E 2$ is saddle. 


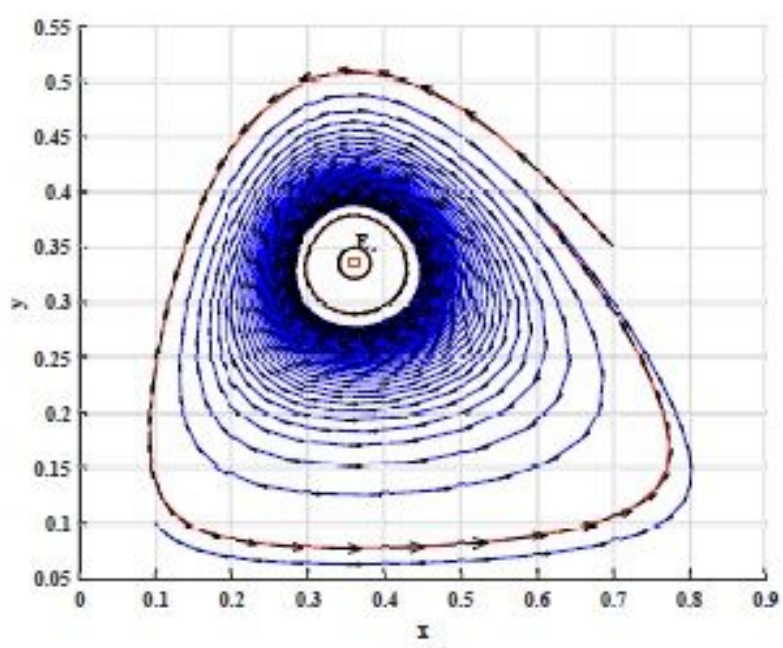

(a)

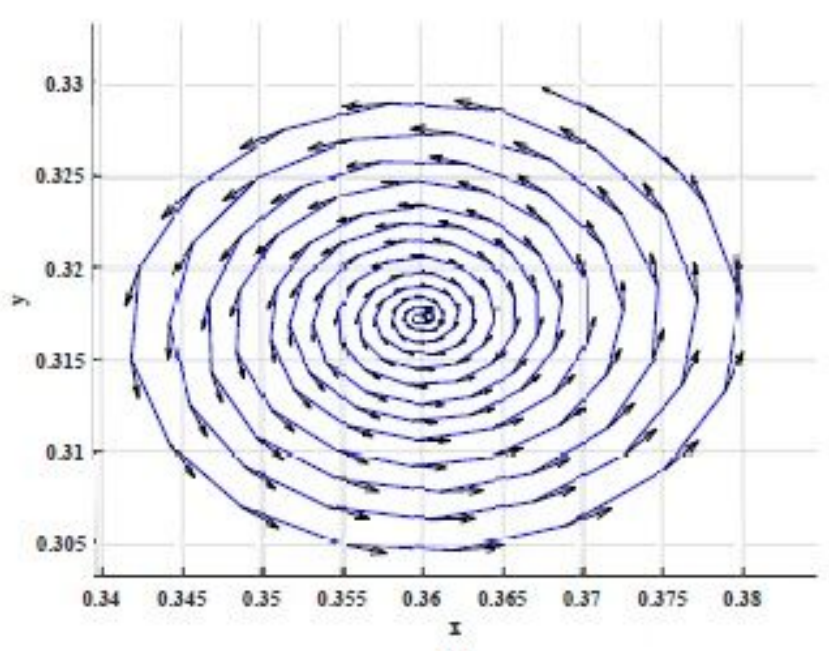

(b)

Figure 2

(a) Phase plot of the system (3) for $c=1 ; h=0: 0288=h 1 ; d=E=0: 3$, where $E^{*}$ is center. (b) Phase plot of the system (3) for $c=1 ; h=0: 0400>h 1 ; d=E=0: 3$, where $E^{\star}$ is source.

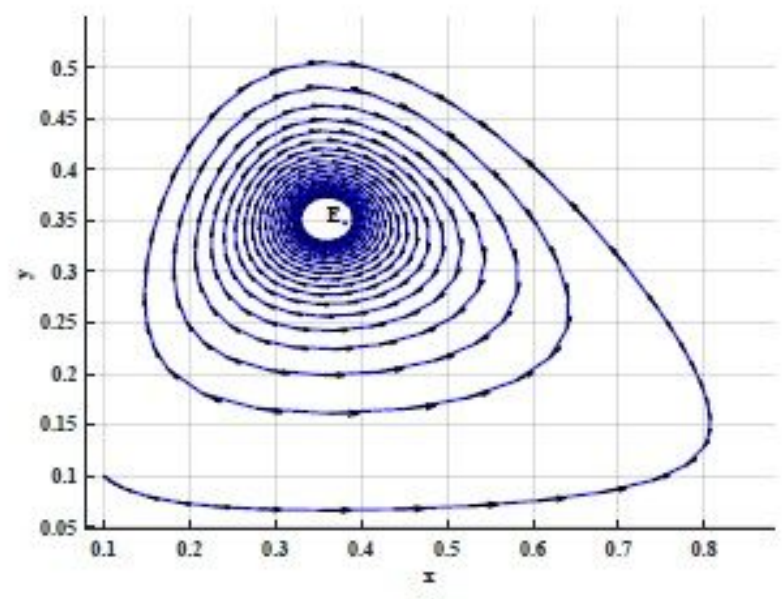

(a)

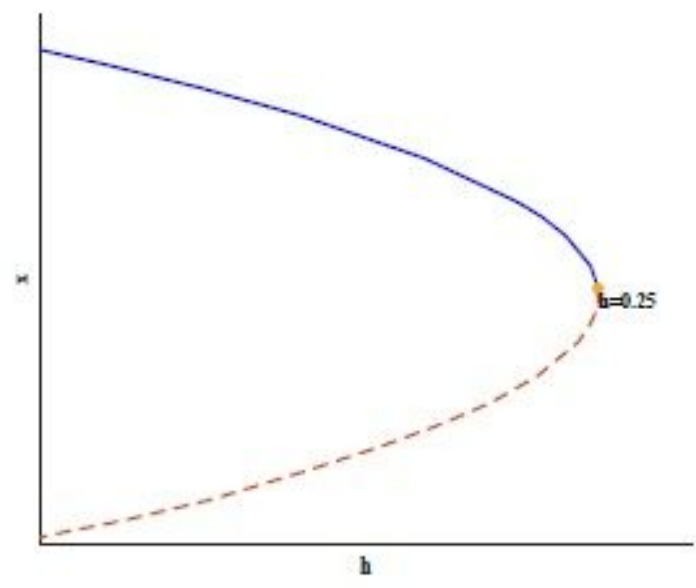

(b)

Figure 3

(a) Phase plot of the system (3) for $c=1 ; h=0: 0288=h 1 ; d=E=0: 3$, where $E^{*}$ is sink. (b) The diagram for saddle-node bifurcation of system (3) at $h=0: 4225$ : Stable equilibrium points are represented by the solid blue curve and unstable equilibrium points are represented by green dashed curve. 


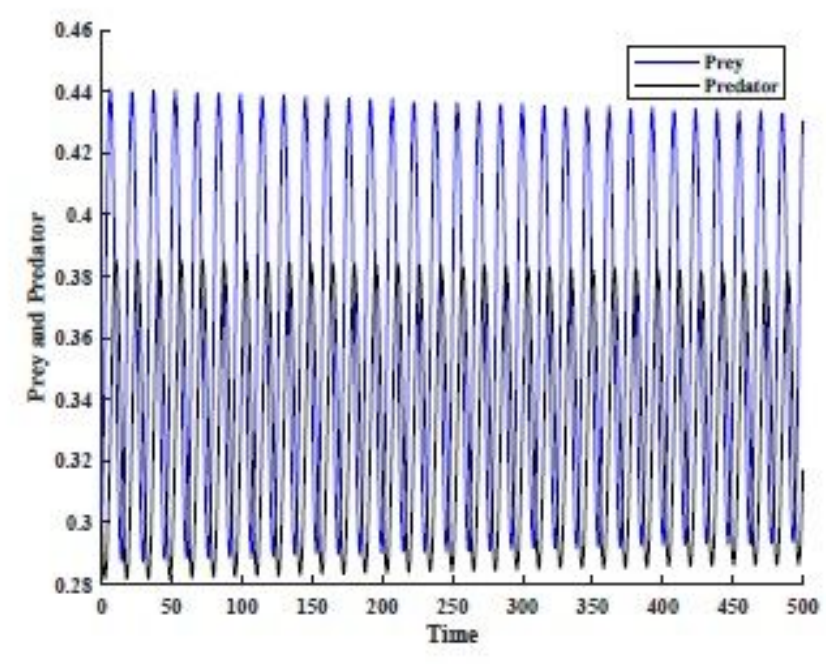

(a)

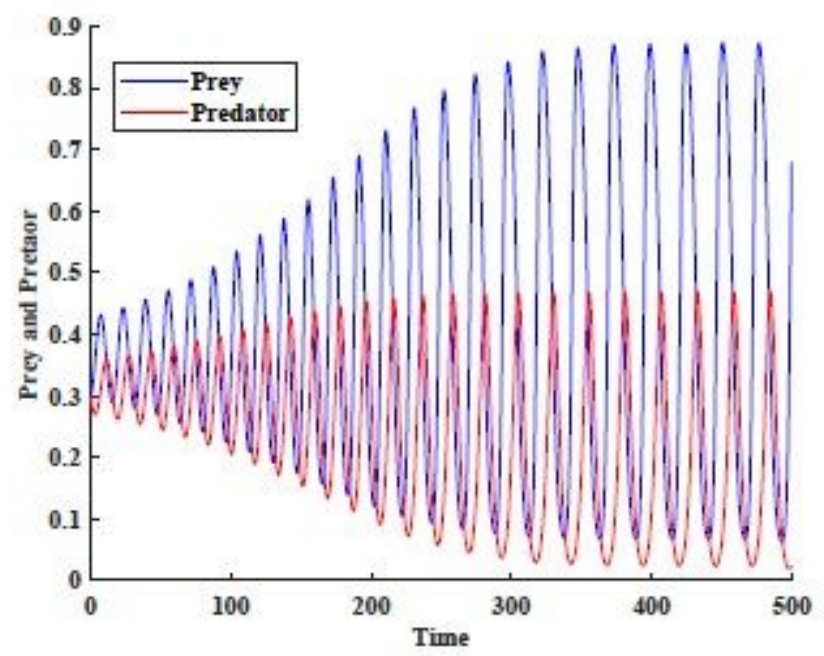

(b)

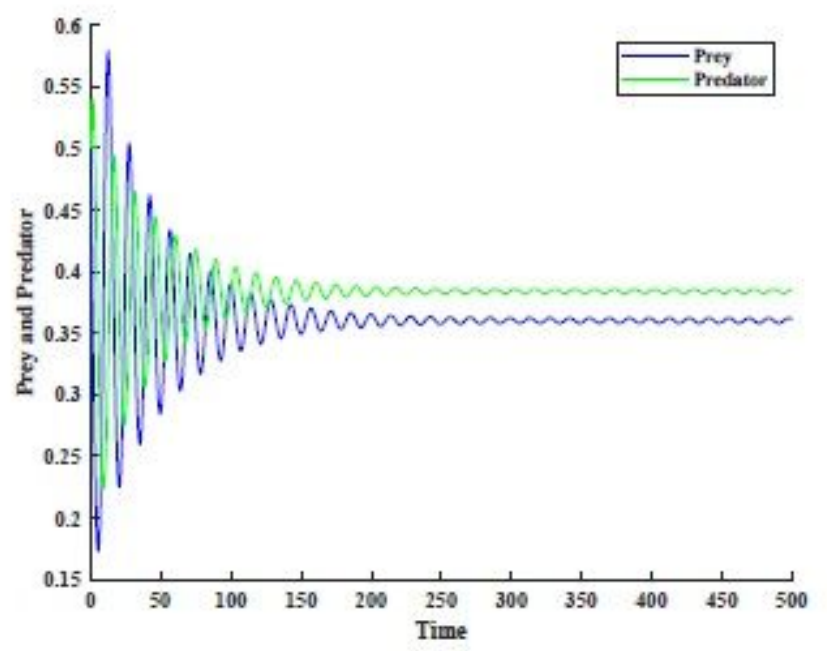

(c)

Figure 4

(a) Time series at $h=0: 0288=h 1$. (b) Time series at $h=0: 0400>h 1$. (c) Time series at $h=0: 0195<h 1$. 